\title{
Squaring operations on truncated polynomial algebras
}

\author{
Dedicated to Professor Atuo Komatu \\ for his 60 -th birthday \\ By \\ Tamio SUGAWARA and Hirosi TODA \\ (Received September 9, 1968)
}

\section{Introduction.}

In algebraic topology there are many important examples of cohomology rings having the structure of a polynomial algebra over Steenrod algebra. It is a basic problem to classify such algebras. In the present paper we discuss the following problem: under what conditions on the set $A$ of the degrees of the variables does a polynomial algebra $P(A)=Z_{2}\left[x_{1}, \cdots \cdots, x_{l}\right]$ admit Steenrod squaring operations? For simplicity of discussions, throughout the paper we assume $A=\left(a_{1}, \cdots \cdots, a_{l}\right)$ is a monotone increasing sequence of positive integers, $i, e_{.}, P(A)=Z_{2}\left[x_{a} ; a \in A\right], \operatorname{deg} x_{a}$ $=a$, has the generators of distinct degrees.

If a polynomial algebra $P(A)$ admits squaring operations, then so does the derived truncated polynomial algebra $T P(A)=P(A) / D^{3}$. We say that a sequence $A$ is allowable if $\operatorname{TP}(A)$ admits squaring operations. A disjoint union $\sum A_{i}$ of allowable sequences $A_{i}$ is again allowable, and we say that $A$ is irreducible if it is not a disjoint union of two non-empty allowable sequences. We denote $2^{s} A=\left(2^{s} a_{j}\right)$ for $A=\left(a_{j}\right)$, then $2^{s} A$ is allowable, if and only if $A$ is so. We say that a sequence $A$ is simple if it is allowable, irreducible and contains an odd integer. It is a direct consequence of the definitions that every allowable sequence $A$ is of the form $\sum 2^{s_{i}} A_{i}$ for some integers $s_{i} \geq 0$ and some simple sequences $A_{i}$.

Our main purpose is to classify the simple sequences, and the result is the following

Main Theorem. The simple sequences are the followings:

$$
\begin{aligned}
& T_{1}=(1), \\
& A_{l}=(2,3, \cdots \cdots, l+1), l \geq 2, l+1 \text { is not a power of } 2, \\
& G_{m}=A_{2}{ }^{m+1}-2-\left(2^{t}+1 ; 0 \leq t \leq m\right), \quad m \geq 2,
\end{aligned}
$$

and $\quad E_{n}=\left(2^{n+1}-2^{s} ; 0 \leq s \leq n\right), n \geq 3$.

Examples of squaring operations on $P(A)$ for $A=T_{1}, A=A_{l}$ and $A=G_{2}=E_{2}=$ $(4,6,7)$ are the cohomology rings of $R P^{\infty}, B S O(l+1)$ and $B G_{2}$ respectively. For other simple sequences we shall give squaring operations on $P(A)$ or $T P(A)$ as subquotient algebras of $H^{*}\left(B S O ; Z_{2}\right)$. As an application we have

Corollary. Let $X$ be an $H$-space. If the cohomology $\operatorname{ring} H^{*}\left(X ; Z_{2}\right)$ is generated by primitive classes $\left\{u_{j}\right\}$ of distinct degrees, then the set $A=\left\{\operatorname{deg} u_{j}+1\right\}$ is a disjoint union of $2^{s i} A_{i}$ for some simple sequences $A_{i}$.

\section{A theorem of Thomas.}

In the following we always assume that a sequence $A=\left(a_{1}, \cdots \cdots, a_{l}\right)$ of integers satisfies 
$(*)$

$$
1 \leq a_{1}<a_{2}<\cdots \cdots<a_{l} .
$$

Denote by

$$
P(A)=Z_{2}\left[x a_{1}, \cdots \ldots, x a_{l}\right], \operatorname{deg} x a_{i}=a_{i},
$$

the polynomial algebra over $Z_{2}$ with the variables $x_{a}, a \in A$. Let $D$ be the positive part of $P(A)$, then $D^{2}=D \cdot D$ consists of the decomposable elements. We denote the derived truncated polynomial algebra by

$$
T P(A)=P(A) / D^{3}, D^{3}=D^{2} \cdot D .
$$

We say that $T P(A)$ (or $P(A)$ ) admits squaring operations if for each integer $n$ there is a linear map $S q^{n}: T P(A) \longrightarrow T P(A)($ or $; P(A) \longrightarrow P(A)$ ) satisfying the following conditions :

(1.1), (i ). $\quad \operatorname{deg}\left(S q^{n} x\right)=\operatorname{deg} x+n, S q^{0}=i d, S q^{n}=0$ for $n<0$.

(ii). (Cartan formula) $S q^{n}(x \cdot y)=\sum_{i+j=n} S q^{i} x \cdot S q^{j} y$.

(iii). (Adem velation) $S q^{a} S q^{b}=\sum_{i}\left(\begin{array}{c}b-i-1 \\ a-2 i\end{array}\right) S q^{a+b-i} S q^{i}$ for $a<2 b$.

(iv). $S q^{n} x=x^{2}$ if $\operatorname{deg} x=n$ and $S q^{n} x=0$ if $\operatorname{deg} x<n$.

Here $\left(\begin{array}{l}a \\ b\end{array}\right)$ stands for the binomial coefficient with the convension: $\left(\begin{array}{l}a \\ b\end{array}\right)=0$ if $a<b$ or if $b<0$.

We say that $A$ is allowable (resp. strictly allowable) if $T P(A)$ (resp. $P(A)$ ) admits squaring operations. By Cartan formula, if $P(A)$ admits squaring operations then $D^{3}$ is closed under the squaring operations, and $T P(A)$ admits squaring operations.

(1.2). If $A$ is strictly allowable then it is allowable.

Thomas has proved a very strong restriction on the behavier of $S q^{n}$ in $T P(A)$ [7; Theorem 1.4]. In our case this is restated as follows.

Theorem 1.1 (Thomas). Let $A$ be an allowable sequence. If $n \in A, k \geq 0$ and $\left(\begin{array}{c}n-k-1 \\ k\end{array}\right) \equiv 1(\bmod 2)$ then $n-k \in A, S q^{k} x_{n-k} \equiv x_{n}$ and $S q^{k} x_{n} \equiv 0 \bmod D^{2}$.

This theorem determines the squaring operations on $T P(A) / D^{2}=P(A) / D^{2}$, that is, with the convention

$$
x_{n}=0 \quad \text { if } \quad n \notin A
$$

we have

Lemma 1.2. $S q^{k} x_{n} \equiv\left(\begin{array}{c}n-1 \\ k\end{array}\right) x_{n+k} \bmod D^{2}$.

Proof. We shall prove by induction on $k$. First consider the case $k=2^{t}$. Then $\left(\begin{array}{c}n-1 \\ 2^{t}\end{array}\right) \equiv 0$ is equivalent to $\left(\begin{array}{c}n-2^{t}-1 \\ 2^{t}\end{array}\right) \equiv 1$, and the lemma follows from the theorem. Next let $k=2^{s+1} d+2^{s}$ for some $d>0$ and $s \geq 0$, and consider Adem relation: $S q^{20} S q^{2^{s+1 d}}$ $=S q^{k}+\sum_{r=0}^{s-1} S q^{k-2^{r}} S q^{2 r}$. We have easily $\left(\begin{array}{c}n-1 \\ 2^{r}\end{array}\right)\left(\begin{array}{c}n+2^{r}-1 \\ k-2^{r}\end{array}\right) \equiv 0$ and $\left(\begin{array}{c}n-1 \\ 2^{s+1} d\end{array}\right)\left(\begin{array}{c}n+2^{s+1} d-1 \\ 2^{s}\end{array}\right)$ $\equiv\left(\begin{array}{c}n-1 \\ k\end{array}\right)$. Then by induction hypothesis we have $S q^{k-2 r} S q^{2 r} x_{n} \equiv 0$ and $S q^{k} x_{n} \equiv S q^{2 s}$ $S q^{2^{s+1}} d x_{n} \equiv\left(\begin{array}{c}n-1 \\ k\end{array}\right) x_{n+k}$.

We shall show various consequences of Thomas' theorem. For $k=1$, we have (1. 4). If $2 m+1 \in A$ then $2 m \in A$ and $S q^{1} x_{2 m} \equiv x_{2 m+1}, S q^{1} x_{2 m+1} \equiv 0 \bmod D^{2}$.

For the simplicity of discussions we choose generators $x_{2 m+1}, m \geq 1$, as follows. (1. 4$)^{\prime}$. $S q^{1} x_{2 m}=x_{2 m+1}$ if $2 m+1 \in A$, thus $S q^{1} x_{2 m+1}=0$ for $m \geq 1$. 
For $k=2$ we have

(1. 5). If $n \equiv 0,3(\bmod 4)$ then $S q^{2} x_{n} \equiv x_{n+2}$ and $n+2 \in A$ implies $n \in A$. If $n \equiv$ $1,2(\bmod 4)$ then $S q^{2} x_{n} \equiv 0$.

Assume that $n=2^{s+1} d+2^{s}$ for $d>0$, then $\left(\begin{array}{c}n-2^{s}-1 \\ 2^{s}\end{array}\right) \equiv 1$, and moreover $\left(\begin{array}{c}n-2^{s+1}-1 \\ 2^{s+1}\end{array}\right)$ $\equiv d-1$. Thus we have the followings.

(1.6). Let $n=2^{s+1} d+2^{s}$. If $d>0$, then $n \in A$ implies $n-2^{s} \in A$. If $d$ is even $>0$, then $n \in A$ implies $n-2^{s+1} \in A$.

(1.7). For an allowable sequence $A=\left(a_{1}, a_{2}, \cdots \cdots, a_{l}\right), a_{1}=2^{t}$ for some $t$, and if $l$ $\geq 2$ then $a_{2} \geq 2^{t}+2^{t-1}$.

As is easily seen

(1.8). If $1 \leq h \leq 2^{t}$ then $2^{t+1}+h \in A$ implies $2^{t}+h \in A$.

Assume that $2^{t}+1<n<2^{t+1}$ and $2^{t+1}-n+1$ is not a power of 2 . Let $2^{t+1}-n+1=$ $2^{s+1} d+2^{s}, d>0$, then $\left(\begin{array}{c}n-1 \\ 2^{s}\end{array}\right) \equiv 1, \quad 2^{t}+1<n+2^{s}<2^{t+1}, \quad$ and $n+2^{s} \in A$ implies $n \in A$. Therefore, if $2^{t+1}-2^{s}+1 \in A$ for $t>s>0$ then $n \in A$ for $2^{t}+1<n<2^{t+1}$ and by (1.8) $2^{t}-2^{s-1}+1 \in A$. Repeating this process we have

(1.9). If $2^{t+1}-2^{s}+1 \in A$ for $t>s>0$ then $i \in A$ for $4 \leq i<2^{t+1}$ and $i \neq 2^{r}+1, r \leq t$, i.e., $G_{t} \subset A$.

Since $\left(\begin{array}{c}2^{t+1}-2^{s} \\ 2^{s}\end{array}\right) \equiv 1$ for $t \geq s \geq 0,2^{t+1}+1 \in A$ implies the assumption of (1.9) and $2^{t}+1,2^{t+1} \in A$, hence $i \in A$ for $2^{t}+1 \leq i \leq 2^{t+1}+1$. Repeating this process we have

(1.10). $2^{t}+1 \in A$ implies $i \in A$ for $2 \leq i \leq 2^{t}+1$, i.e., $A_{2^{t}} \subset A$.

Since $\left(\begin{array}{c}2^{t}-2 \\ k\end{array}\right) \equiv 1$ for even $k$ of $2^{t}-1>k>0$, we have

(1.11). If $n$ is odd, $n \in A$ and $2^{t+1}-1>n>2^{t}$ then $2^{t}-1 \in A$.

(1.12). $n \in A$ and $2^{t+1}>n>2^{t}$ imply $2^{t} \in A$.

Finally we see that Thomas' theorem determines the squaring operations in $D^{2} \subset T P(A)$.

(1.13). $S q^{k}\left(x_{m} x_{n}\right)=\sum_{i+j=k} S q^{i} x_{m} \cdot S q^{j} x_{n}=\sum_{i+j=k}\left(\begin{array}{c}m-1 \\ i\end{array}\right)\left(\begin{array}{c}n-1 \\ j\end{array}\right) x_{m+i} x_{n+j}$ in TP(A).

\section{Reduction to simple cases.}

Let a sequence $A$ be a disjoint union of two allowable sequences $B$ and $C$. As the tensor product over mod 2 Steenrod algebra (Hopf algebra), $T P(B) \otimes T P(C)$ admits squaring operations, and $T P(A)=(T P(B) \otimes T P(C)) / D^{3}$ does so. Thus

(2.1). If $B$ and $C$ are allowable and $B \cap C=\phi$, then $A=B+C$ is allowable.

The notation $B<C($ resp. $B \leq C)$ means that $b<c($ resp. $b \leq c)$ for any $b \in B$ and $c \in C$.

Lemma 2.1. If $A=B+C$ is allowable, $B<\left(2^{t}\right) \leq C$ and $n \notin C$ for $2^{t}<n<2^{t}+2^{\ell-1}$, then $B$ and $C$ are both allowable.

Proof. Consider squaring operations on $T P(A)=Z_{2}\left[x_{a} ; a \in A\right] / D^{3}$. Let $I_{B}$ and $I_{C}$ be the ideals of $T P(A)$ generated by $\left\{x_{b} ; b \in B\right\}$ and $\left\{x_{c} ; c \in C\right\}$ respectively. By (1.1), (iv), $S q^{2 r} x_{b}=0$ for $r \geq t, b \in B$. Let $r<t$, then $2^{r}+b<2^{t}+2^{t-1}$. Thus $S q^{2 r} x_{b} \in I_{B}$

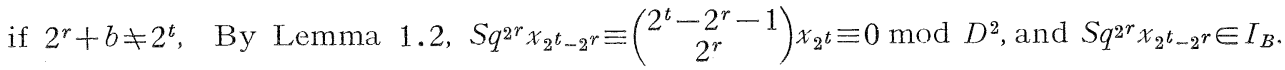
Since the operations $S q^{2 r}, r \geq 0$ generate the squaring operations we have proved 
that $I_{B}$ is closed under the squaring operations. The ideal $I_{B} \cap I_{C}$ is spanned by $\left\{x_{b} x_{c}\right\}$. Then (1.13) shows that $I_{B} \cap I_{C}$ is closed under the squaring operations. Consequently $T P(A) / I_{B}=T P(C)$ and $I_{B} /\left(I_{B} \cap I_{C}\right)=T P(B)$ admit squaring operations, hence $B$ and $C$ are allowable.

We have defined that an allowable sequence is irreducible if it is not a disjoint union of two non-empty allowable sequences. Obviously an allowable sequence is a disjoint union $A=\sum A_{i}$ of irreducible sequences $A_{i}$. Let $B$ and $C$ be disjoint irreducible sequences whose minimum numbers are $b_{1}=2^{s}$ and $c_{1}=2^{t}$ for some $s, t$, by (1.7). We may assume $s<t$. By (1.12), any $b \in B$ satisfies $b<2^{t}$ or $b \geq 2^{t+1}$.

Lemma 2.1 shows that there is no element of $b \in B$ with $b \geq 2^{t+1}$. Thus $B<C$, and we have

Theorem 2.2. Any allowable sequence $A$ is decomposed (uniquely) into a disjoint union $A=\sum A_{i}$ of irreducible sequences $A_{i}$ satisfying $A_{i}<A_{j}$ for $i<j$.

For each sequence $A=\left(a_{1}, a_{2}, \cdots \ldots, a_{l}\right)$ and each integer $s \geq 0$, we define

$$
2^{s} A=\left(2^{s} a_{1}, 2^{s} a_{2}, \cdots \cdots, 2^{s} a_{l}\right)
$$

and also

$$
A / 2^{s}=\left(b_{1}, \cdots \cdots, b_{k}\right)
$$

by that $b \in A / 2^{s}$ if and only if $2^{s} b \in A$. Let $A$ be allowable and $T P(A)=Z_{2}\left[x_{a}\right] / D^{3}$ admit squaring operations. Define ring homomorphisms (epimorphisms) $\theta: T P(A) \rightarrow$ $T P(2 A)=Z_{2}\left[y_{2 a}\right] / D^{3}$ and $\lambda: T P(A) \longrightarrow T P(A / 2)=Z_{2}\left[z_{b}\right] / D^{3}$ by $\theta\left(x_{a}\right)=y_{2 a}, \lambda\left(x_{2 b}\right)=z_{b}$ and $\lambda\left(x_{a}\right)=0$ for odd $a$. Then it is directly checked that $T P(2 A)$ and $T P(A / 2)$ admit squaring operations by putting $S q^{2 k} y_{2 a}=\theta\left(S q^{k} x_{a}\right), S q^{2 k-1} y_{2 a}=0$ and $S q^{k} z_{b}=\lambda\left(S q^{2 k} x_{2 b}\right)$. The same is true for $P(2 A)$ and $P(A / 2)$. Since $(2 A) / 2=A$ we have

(2.2). A is (strictly) allowable if and only if $2^{\mathrm{s}} A$ is (strictly) allowable. If $A$ is (strictly) allowable then so is $A / 2^{s}$.

We say that an allowable sequence is simple if it is irreducible and contains an odd integer. Obviously any irreducible sequence is of a form $2^{s} A^{\prime}$ for some simple sequence $A^{\prime}$. Thus we have

Corollary. Any allowable sequence is a disjoint union $A=\sum 2^{s_{i}} A_{i}$ for some simple sequences $A_{i}$.

We shall use the following lemmas.

Lemma 2.3. An allowable sequence $A=\left(a_{1}, \ldots \ldots, a_{l}\right)$ is irreducible if and only if the following condition is satisfied: if $2^{t} \in A, 2^{t}>a_{1}$, then there exists an $n \in A$ such that $2^{t}<n<2^{t}+2^{t-1}$.

Proof. If $A$ is not irreducible, then by Theorem $2.2 A=B+C$ for some allowable $B$ and $C$ with $B<C$. By (1.7), $C=\left(2^{t}, \ldots \ldots\right)$ and $n \notin C$ for $2^{t}<n<2^{t}+2^{t-1}$, and the condition is not satisfied. Conversely, if the condition is not satisfied then $A$ is not irreducible by Lemma 2.1 .

Lemma 2.4. If $A$ is simple and $2 \notin A$, then $A / 2$ is also simple or empty.

Proof. By (2.2), $A / 2$ is allowable. Consider $2^{t} \in A / 2$ which is not minimum. Then $2^{t+1} \in A$ and it is not minimum. By Lemma 2.3 there exists an $m \in A$ such that $2^{t+1}<m<2^{t+1}+2^{t}$. If $m=2 n$ then $n \in A / 2$ and $2^{t}<n<2^{t}+2^{t-1}$. Since $2 \notin A$, 
$2^{t+1}+1 \notin A$ by (1.10). Thus, if $m=2 n+1$ then $2^{t}<n<2^{t}+2^{t-1}, 2 n \in A$ by (1.4), and $n \in A / 2$. It follows from Lemma 2.3 that $A / 2$ is irreducible. If $1 \in A$ then the irreducibility implies $A=(1)$, whence $A / 2=\phi$. By (1.10), $3,5 \notin A$. So, we may assume that $A$ contains an odd integer $2 n+1 \geq 7$, whence $n \in A / 2$. If $n$ is odd this shows that $A / 2$ is simple. If $n$ is even then $2 n+1 \in A$ implies $2 n-1 \in A$ by (1.5) and hence $n-1 \in A / 2$. Thus $A / 2$ is simple or empty.

\section{A lemma.}

The following lemma is a key to the discussions in the subsequent sections. If $n+1 \in A$ and $2 n+1 \notin A$ for an allowable sequence $A$, then $S q^{n} x_{n+1}$ is decomposable, i.e., we can write down

$$
S q^{n} x_{n+1}=\sum a_{j} x_{n-j} x_{n+1+j} \text { for some } a_{j} \in Z_{2}, j \geq 0 \text {. }
$$

Lemma 3.1. Assume that $i \in A$ for $n-k \leq i \leq n+k+1, k \geq 0$, and $2 n+1 \notin A$. Then the coefficients $a_{j}$ in the expansion (3.1) of $S q^{n} x_{n+1}$ satisfy

$a_{j}=1$ for $0 \leq j \leq k$ if $n+k$ is even or if $n$ is even and $n+k \equiv 1(\bmod 4)$,

$a_{j}=1$ for $0 \leq j \leq k-1$ if $n+k \equiv 3(\bmod 4)$ or if $n$ and $n+k$ are odd.

Proof. Case 1, $n$ : even. First consider the case that $n$ is even. We have

$$
S q^{1} S q^{n} x_{n+1}=S q^{n+1} x_{n+1}=x^{2}{ }_{n+1}
$$

and

$$
S q^{2} S q^{n} x_{n+1}=(n / 2+1) S q^{n+2} x_{n+1}+S q^{n+1} S q^{1} x_{n+1}=0
$$

by (1.1) and $\left(1.4^{\prime}\right)$. Applying $S q^{1}$ and $S q^{2}$ to (3.1) we have, by (1.13), (1.4) and (1.5),

$$
x_{n+1}^{2}=S q^{1} S q^{n} x_{n+1}=a_{0} x_{n+1}^{2}+\sum_{s \geq 1}\left(a_{2 s-1}+a_{2 s}\right) x_{n-2 s+1} x_{n+2 s+1},
$$

when $n \equiv 0(\bmod 4) \quad 0=S q^{2} S q^{n} x_{n+1}=\left(a_{0}+a_{1}\right) x_{n+1} x_{n+2}+\sum_{t \geq 0}\left\{\left(a_{4 t+2}+a_{4 t+4}\right) x_{n-4 t-2} x_{n+4 t+5}\right.$

and when $n \equiv 2(\bmod 4)$

$$
\left.+\left(a_{4 t+3}+a_{4 t+5}\right) x_{n-4 t-3} x_{n+4 t+6}\right\}
$$

$$
0=S q^{2} S q^{n} x_{n+1}=\sum_{t \geq 0}\left\{\left(a_{4 t}+a_{4 t+2}\right) x_{n-4} x_{n+4 t+3}+\left(a_{4 t+1}+a_{4 t+3}\right) x_{n-4 t-1} x_{n+4 t+4}\right\} .
$$

Thus we have the following sequences of equalities up to $j \leq k$.

$$
\begin{aligned}
& 1=a_{0}=a_{1}=a_{2}=\cdots \cdots=a_{4 t+2}=a_{4 t+4}=a_{4 t+3}=a_{4 t+5}=a_{4 t+6}=\cdots \cdots \quad(n \equiv 0), \\
& 1=a_{0}=a_{2}=a_{1}=\cdots \cdots=a_{4 t}=a_{4 t+2}=a_{4 t+1}=a_{4 t+3}=a_{4 t+4}=\cdots \cdots \quad(n \equiv 2) .
\end{aligned}
$$

It follows that $a_{j}=1$ for $0 \leq j \leq k$ if $n+k \neq 3(\bmod 4)$ and $a_{j}=1$ for $0 \leq j \leq k-1$ if $n+$ $k \equiv 3(\bmod 4)$.

Case 2, $n$ : odd. Next consider the case that $n$ is odd. By (1.5) $2 n+1 \notin A$ implies $2 n+3 \notin A$. By what we have just proved, in

$$
S q^{n+1} x_{n+2}=\sum_{i \geq 0} b_{i} x_{n+1-i} x_{n+2+i}
$$

$$
b_{i}=1 \text { for } 0 \leq i<k \text { when } n+k \neq 2(\bmod 4)
$$

and $\quad b_{i}=1$ for $0 \leq i<k-1$ when $n+k \equiv 2(\bmod 4)$.

From the relation $0=S q^{1} S q^{n} x_{n+1}=\sum_{s \geq 0}\left(a_{2 s}+a_{2 s+1}\right) x_{n-2 s} x_{n+2 s+2}$, it follows

$\left.{ }^{*}\right) a_{2 s}=a_{2 s+1}$ for $0 \leq s \leq(k-1) / 2$.

Also we have when $n \equiv 1(\bmod 4)$

$$
S q^{2} S q^{n} x_{n+1}=\sum_{t \geq 0}\left\{a_{4 t+1}\left(x_{n-4 t+1} x_{n+4 t+2}+x_{n-4 t-1} x_{n+4 t+4}\right)\right.
$$


and when $n \equiv 3(\bmod 4)$

$$
\left.+a_{4 t+2}\left(x_{n-4 t} x_{n+4 t+3}+x_{n-4 t-2} x_{n+4 t+5}\right)\right\}
$$

$$
\begin{aligned}
S q^{2} S q^{n} x_{n+1} & =\sum_{t \geq 0}\left\{a_{4 t}\left(x_{n-4 t+2} x_{n+4 t+1}+x_{n-4}^{\prime} x_{n+4 t+3}\right)\right. \\
& \left.+a_{4 t+3}\left(x_{n-4 t-1} x_{n+4 t+4}+x_{n-4 t-3} x_{n+4 t+6}\right)\right\} .
\end{aligned}
$$

In the equality $S q^{2} S q^{n} x_{n+1}=((n-1) / 2) S q^{n+2} x_{n+1}+S q^{n+1} S q^{1} x_{n+1}=S q^{n+1} x_{n+2}$, comparing the coefficients we have when $n \equiv 1(\bmod 4)$

$a_{4 t+1}=b_{4 t}=1$ for $4 t \leq k-1$ and $a_{4 t+2}=b_{4 t+1}=1$ for $4 t+1 \leq k-2$,

and when $n \equiv 3(\bmod 4)$

$a_{4 t}=b_{4 t-1}=1$ for $4 t-1 \leq k-2$ and $a_{4 t+3}=b_{4 t+2}=1$ for $4 \cdot t+2 \leq k-1$.

Combining with (*) we have that $a_{j}=1$ for $0 \leq j \leq k$ if $n+k$ is even and $a_{j}=1$ for $0 \leq j \leq k-1$ if $n+k$ is odd.

The proof of the lemma has been established.

We shall use the following cases of Lemma $3.1(t \geq 2)$.

(3.2). $n=2^{t}, k=1 ; S q^{2 t} x_{2^{t}+1}=x_{2^{t}-1} x_{2^{t}+2}+$ other terms.

(3.3). $2^{t-1}+1 \leq n<2^{t}-1, n+k+1=2^{t}+1 ; S q^{n} x_{n+1}=x_{2 n+1-2_{-}} x_{2} t+$ other terms.

(3.4). $n$ is even, $k=0 ; S q^{n} x_{n+1}=x_{n} x_{n+1}+$ other terms.

(3.5). $n=2^{t}, k=2^{t}-2 ; S q^{2 t} x_{2} t_{+1}=x_{2} x_{2}{ }^{t+1}-1+$ other terms.

The following special modification of the lemma will be used.

(3.6). If $2^{t}+2^{t-1}-1 \in A$ and $2^{t+1}-1 \notin A, t \geq 2$, then $S q^{2 t-1} x_{2} t=x_{2^{t}-1} x_{2} t+o t h e r$ terms.

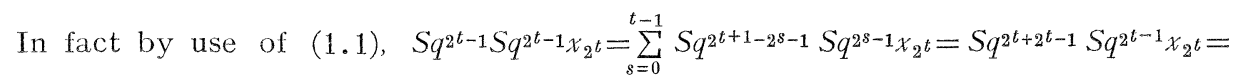
$S q^{2 t+2 t-1-1}\left(x_{2^{t}+2^{t-1}-1}+\right.$ decomposable terms $)=x^{2}{ }^{t}{ }^{t}+2^{t-1}-1$. Assume $x^{2} 2^{t}+2^{t-1}-1=S q^{2 t-1+j} x_{2^{t}-j-1}$ $S q^{2^{t-1-j-1}} x_{2^{t}+j}=\left(\begin{array}{c}2^{t}-j-2 \\ 2^{t-1}+j\end{array}\right)\left(\begin{array}{c}2^{t}+j-1 \\ 2^{t-1}-j-1\end{array}\right) x^{2}{ }_{2^{t}+2^{t-1}-1}$ by (1.13). If $j>0$, then $0 \neq\left(\begin{array}{c}2^{t}+j-1 \\ 2^{t-1}-j-1\end{array}\right)$ $\equiv\left(\begin{array}{c}j-1 \\ 2^{t-1}-j-1\end{array}\right)$ implies $j-1 \geq 2^{t-1}-j-1,2^{t-1}+j \geq 2^{t}-j>2^{t}-j-2$ and hence $\left(\begin{array}{c}2^{t}-j-2 \\ 2^{t-1}+j\end{array}\right)$

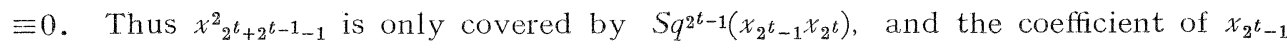
$x_{2} t$ must be 1 modulo 2 .

\section{Classical cases.}

The purpose of this section is to prove the following

Theorem 4.1. If a simple sequence $A$ contains $2 \in A$, then

$$
A=A_{l}=(2,3, \cdots, l+1)
$$

for some $l+1$ which is not a power of 2 .

First consider $S q^{2 a} S q^{n} x_{n+1}$. If $a>n$ it is trivial by (1.1), (iv). Let $a<n$. By (iii) and (iv) of (1.1) we have $S q^{2 a} S q^{n} x_{n+1}=S q^{a+n} S q^{a} x_{n+1}$. Now assume $\left(\begin{array}{l}n \\ a\end{array}\right) \equiv 0$, then $S q^{a} \chi_{n+1}$ is decomposable. It follows easily from (ii) and (iv) of (1.1) that $S q^{a+n} S q^{a} x_{n+1}=0$ (in $T P(A)$ ). Similar arguments hold if $a+n+1 \notin A$, and we have (4.1). If $\left(\begin{array}{l}n \\ a\end{array}\right) \equiv 0(\bmod 2)$ or if $a+n+1 \notin A$ then $S q^{2 a} S q^{n} x_{n+1}=0$.

Lemma 4.2. Let $t \geq 2$ and $A$ be an allowable sequence. If $2^{t}+1 \in A$ and $2^{t+1}+2$ $\in A$ then $2^{t+1}+1 \in A$.

Proof. By (1.10) and (1.8) the assumption implies $i \in A$ for $2 \leq i \leq 2^{t}+2$. Assume 
that $2^{t+1}+1 \notin A$, then we can apply Lemma 3.1 for the case (3.2), and $S q^{2} x_{2^{t}+1}=$ $x_{2}{ }_{-1} x_{2} t_{+2}+$ other terms. To this equality apply $S q^{2 t}$. Since $\left(\begin{array}{c}2^{t} \\ 2^{t-1}\end{array}\right) \equiv 0, S q^{2 t} S q^{2 t} x_{2^{t}+1}=0$ by (4.1). Thus $0=S q^{2}\left(\sum_{j \geq 0} a_{j} x_{2} t_{-j} x_{2} t+1+j\right)$ where $a_{1}=1$. Comparing the coefficients in $x_{2} t_{-1} x_{2} t+1+2$ we have

$$
0 \equiv a_{1}+\sum_{j \geq 2} a_{j}\left(\begin{array}{c}
2^{t}-j-1 \\
j-1
\end{array}\right)\left(\begin{array}{c}
2^{t}+j \\
2^{t}-j+1
\end{array}\right) \quad(\bmod 2) .
$$

Let $j \geq 2$, then $0 \neq\left(\begin{array}{c}2^{t}+j \\ 2^{t}-j+1\end{array}\right) \equiv\left(\begin{array}{c}j \\ 2^{t}-j+1\end{array}\right)$ implies $j \geq 2^{t}-j+1, j-1>2^{t}-j-1$ and hence $\left(\begin{array}{c}2^{t}-j-1 \\ j-1\end{array}\right) \equiv 0$. It follows a contradiction $0 \equiv a_{1}=1(\bmod 2)$. Therefore we obtain $2^{t+1}+1 \in A$.

Lemma 4.3. Let $2 \leq m<2^{t+1}$ and $A$ be allowable. If $2^{t}+1 \in A$ and $m \in A$ then $i \in$ A for $2 \leq i \leq m$.

Proof. We prove by induction on $m$. For the case $m \leq 2^{t}+2$ the lemma is obvious by (1.10). If $m$ is odd then $m-1 \in A$ by (1.4) and the lemma is true by the induction hypothesis. So, it is sufficient to prove that $m=2 n+2 \in A, 2^{t-1}<n<$ $2^{t}-1$, and $2^{t}+1 \in A$ imply $2 n+1 \in A$. Assume that $2 n+1 \notin A$, then by Lemma 3.1 for the case (3.3) we have $a_{2} t_{-n-1}=1$ in $S q^{n} x_{n+1}=\sum a_{j} x_{n-j} x_{n+1+j}$. Since $2^{t-1}<n<$ $2^{t}-1, \quad\left(\begin{array}{c}n \\ n+1-2^{t-1}\end{array}\right)=\left(\begin{array}{c}n \\ 2^{t-1}-1\end{array}\right) \equiv 0 . \quad$ By (4.1) it follows $0=S q^{2 n+2-2 t} S q^{n} x_{n+1}$

$=\sum a_{j} S q^{2 n+2-2 t}\left(x_{n-j} x_{n+1+j}\right)$, and compairing the coefficients in $x_{2^{n-2}{ }^{t}+1^{2}} x_{2+2}$

$$
0 \equiv \sum_{j \geq 0} a_{j}\left(\begin{array}{c}
n-j-1 \\
n+j-2^{t}+1
\end{array}\right)\left(\begin{array}{c}
n+j \\
n-j+1
\end{array}\right) \quad(\bmod 2) .
$$

$\left(\begin{array}{c}n-j-1 \\ n+j-2^{t}+1\end{array}\right) \neq 0$ implies $n+j \geq 2^{t}-1$ and $n-j-1 \geq n+j-2^{t}+1$. If $n+j=2^{t}-1$ then $\left(\begin{array}{c}n-j-1 \\ n+j-2^{t}+1\end{array}\right)\left(\begin{array}{c}n+j \\ n-j+1\end{array}\right) \equiv 1$. If $n+j \geq 2^{t}$ then $\left(\begin{array}{c}n+j \\ n-j+1\end{array}\right) \equiv\left(\begin{array}{c}n+j-2^{t} \\ n-j+1\end{array}\right) \equiv 0$ since $n+j-2^{t}$ $<n+j-2^{t}+1 \leq n-j-1<n-j+1$. Thus we have a contradiction $0 \equiv a_{2^{t}-n-1} \equiv 1$ (mod 2). Therefore we obtain $2 n+1 \in A$.

Proof of Theorem 4.1. If $1 \in A$ then $A$ is not irreducible by Lemma 2.3. Thus $1 \notin A$. If $3 \notin A$ then $A=(2)+(A-(2))$ is not simple by Lemma 2.1. Thus $3 \in A$. Let $t$ be the maximal number such that $2^{t}+1 \in A$, and $2^{t+1}+1 \notin A$, and let $a_{l}$ be the maximal number of $A$. If $a_{l}<2^{t+1}$ then it follows from Lemma $4.3 A=A_{l}$. Now assume $a_{l} \geq 2^{t+1}$ and lead to a contradiction. If $2^{t+1} \notin A$ then $i \notin A$ for $2^{t+1}<i$ $<2^{t+2}$ by (1.12), and $A$ is not irreducible by Lemma 2.1. Thus $2^{t+1} \in A$. By Lemma 2.3 there exists an $n \in A$ such that $2^{t+1}<n<2^{t+1}+2^{t}$. Let $n_{0}=2^{t+1}+2^{s}(2 a+1)$ be the minimal of such $n$. By (1.6), $n_{0} \in A$ implies $n_{0}-2^{s} \in A$. By the choice of $n_{0} a$ must be zero and $n_{0}=2^{t+1}+2^{s}$. Since $2^{t+1}+1 \notin A, t>s \geq 1$. By (1.6), $2^{t+1}+2^{s} \in A$ implies $2^{t+1}-2^{s} \in A$. Then applying Lemma 4.3 we have $i \in A$ for $2 \leq i \leq 2^{t+1}-2^{s}$, in particular $2^{t}+2^{s-1} \in A$. Now consider the allowable sequence $A / 2^{s-1}$. Then $2^{t-s+1}$ $\in A / 2^{s-1}$ and $2^{t-s+2}+2 \in A / 2^{s-1}$. It follows from Lemma 4.2 that $2^{t-s+2}+1 \in A / 2^{s-1}$. Since $2^{s-1}\left(A / 2^{s-1}\right) \subset A$ we have $2^{t+1}+2^{s-1} \in A$ which contradicts to the minimum condition of $n_{0}=2^{t+1}+2^{s}$. We have proved the theorem. 


\section{Exceptional cases.}

The purpose of this section is to prove the following theorems.

Theorem 5.1. If a simple sequence $A$ satisfies $2 \notin A$ and $4 \in A$ then, for some $m \geq 2, A=G_{m}=A_{2^{m+1}-2}-\left(2^{t}+1 ; 0 \leq t \leq m\right)=\left(i ; 4 \leq i \leq 2^{m+1}-1, i-1\right.$ is not a power of 2$)$.

Theorem 5.2. If a simple sequence $A$ does not contain 1,2 and 4 then, for some $n \geq 3, A=E_{n}=\left(2^{n+1}-2^{s} ; 0 \leq s \leq n\right)=\left(2^{n}, 2^{n}+2^{n-1}, \cdots \cdots, 2^{n+1}-2,2^{n+1}-1\right)$.

Obviously, if a simple sequence contains 1 then $A=T_{1}=(1)$. This and Theorems $4.1,5.1,5.2$, cover all the cases of simple sequences.

We prepare the following lemmas.

Lemma 5.3. Let $t>s+1$ and $s>0$. If an allowable sequence $A$ satisfies $2 \notin A$, $2^{t}-2^{s}+1 \in A$ and $2^{t}+2^{s} \in A$ then $2^{t+1}-2^{s+1}+1 \in A$.

Proof. Assume $2^{t+1}-2^{s+1}+1 \notin A$ then by Lemma 3.1 of the case (3.4) $S q^{n} x_{n+1}$ $=x_{n} x_{n+1}+\cdots \cdots$ for $n=2^{t}-2^{s}$. Since $2 \notin A, 2^{s}+n+1=2^{t}+1 \notin A$ by (1.10). It follows from (4.1) $0=S q^{2 s+1} S q^{n} x_{n+1}=S q^{2^{s+1}} \sum a_{j} x_{n-j} x_{n+1+j}$. Consider the coefficients in $x_{2}{ }^{t}+2^{s} x_{2} t_{-2} s+1$ then we have

$$
0 \equiv a_{0}\left(\begin{array}{c}
2^{t}-2^{s}-1 \\
2^{s+1}
\end{array}\right)+\sum_{j \geq 1} a_{j}\left(\begin{array}{c}
2^{t}-2^{s}-j-1 \\
j+1
\end{array}\right)\left(\begin{array}{c}
2^{t}-2^{s}+j \\
2^{s+1}-j-1
\end{array}\right) \quad(\bmod 2)
$$

where $a_{0}=1$. Since $t>s+1,\left(\begin{array}{c}2^{t}-2^{s}-1 \\ 2^{s+1}\end{array}\right) \equiv 1 . \quad$ Let $j \geq 1$ and assume $\left(\begin{array}{l}2^{t}-2^{s}+j \\ 2^{s+1}-j-1\end{array}\right) \equiv 1$ then $j+1 \leq 2^{s+1}$. Consider the case $j+1 \neq 2^{s}, \quad 2^{s+1}$ then $j+1=2^{r}(2 a+1), \quad r<s$, and $\left(\begin{array}{c}2^{t}-2^{s}+j \\ 2^{s+1}-j-1\end{array}\right)=\left(\begin{array}{c}2^{t}-2^{s}+2^{r+1} a+2^{r}-1 \\ 2^{s+1}-2^{r+1}(a+1)+2^{r}\end{array}\right) \equiv 0 . \quad$ If $j+1=2^{s}$ then $\left(\begin{array}{c}2^{t}-2^{s}-j-1 \\ j+1\end{array}\right)=\left(\begin{array}{c}2^{t} \\ 2^{s}\end{array}\right) \equiv 0 . \quad$ If $j+1=2^{s+1}$ then $\left(\begin{array}{c}2^{t}-2^{s}-j-1 \\ j+1\end{array}\right)=\left(\begin{array}{c}2^{t}-2^{s+2}+2^{s} \\ 2^{s+1}\end{array}\right) \equiv 0 . \quad$ Therefore we have a contradiction $0 \equiv a_{0} \equiv 1(\bmod 2)$, and $2^{t+1}-2^{s+1}+1$ must be contained in $A$.

Lemma 5.4. Let $t \geq 3$. If an allowable sequence $A$ satisfies $2 \notin A, 2^{t}+2 \in A$ and $2^{t+1}-3 \in A$ then $2^{t+1}-1 \in A$.

Proof. $2 \notin A$ implies $2^{t}+1 \notin A$. Since $\left(\begin{array}{c}2^{t}+2^{t-1}-2 \\ 2^{t-1}-2\end{array}\right) \equiv 1,2^{t+1}-3 \in A$ implies $2^{t}+$ $2^{t-1}-1 \in A$. Now assume that $2^{t+1}-1 \notin A$, then by (3.6) we have $S q^{2^{t}-1} x_{2^{t}}=x_{2^{t}-1} x_{2^{t}}$ $+a_{2} x_{2} t_{-3} x_{2} t_{+2}+\cdots \cdots$ By use of (1.5) Sq $S q^{2}{ }^{t-1} x_{2^{t}}=x_{2^{t}-1} x_{2^{t}+2}+$ other terms. On the other hand we have by (4.1) $S q^{2} S q^{2 t-1} x_{2} t=0$ since $2^{t+1}+1 \notin A$. This is a contradiction and we conclude $2^{t+1}-1 \in A$.

Lemma 5.5. Let $t \geq 2$. If an allowable sequence $A$ satisfies $2 \notin A, 2^{t}+2 \in A$ and $2^{t+1}-1 \in A$ then $2^{t}+3 \in A$ or $2^{t+1}+2 \in A$.

Proof. Assume that $2^{t}+3 \notin A$ and $2^{t+1}+2 \in A . \quad 2^{t+1}-1 \in A$ implies $2^{t+1}-2 \in A$. Then $A / 2$ is allowable and satisfies $2^{t-1}+1 \in A / 2,2^{t}-1 \in A / 2$ and $2^{t}+1 \notin A / 2$. By Lemma $4.3, i \in A / 2$ for $2 \leq i \leq 2^{t}-1$. Then we can apply Lemma 3.1 for the case 
(3.5) and we have $S q^{2 t-1} z_{2^{t-1}+1}=z_{2} z_{2} t_{-1}$ tother terms in $T P(A / 2)$. Passing to $A$, we have $S q^{2 t} x_{2^{t}+2}=x_{4} x_{2} t_{+1}$ tother terms. Thus $S q^{2 t+1} x_{2} t_{+2}=x_{4} x_{2^{t+1}-1}$ tother terms. By Adem relation $S q^{2}{ }^{2 t+1} x_{2} t+2=S q^{2} S q^{2 t-1} x_{2^{t}+2}+S q^{2 t} S q^{1} x_{2} t+2$. Since $2 \notin A$ we have $2^{t+1}+1 \notin$ $A$ and $S q^{2 t-1} x_{2} t+2$ is decomposable. By the assumption $2^{t}+3 \notin A, S q^{1} x_{2^{t}+2}$ is decomposable. Since $x_{4} \equiv S q^{k} x_{4-k}$ holds only for $k=0$, the possibility to cover $x_{4} x_{2} 2^{t+1}-1$ by $S q^{2} D^{2}+S q^{2 t} D^{2}$ is $x_{4} S q^{2} x_{2}{ }^{t+1-3}$ and $x_{4} S q^{2 t} x_{2} t_{-1}$ both of which are trivial by (1.5) and (1.1), (iv). So, we arrive to a contradiction. Thus $2^{t}+3 \in A$ or $2^{t+1}+2 \in A$.

Proof of Theorem 5.1 .

Case $1: 7 \in A$. Obviousiy $1 \notin A$. Since $2 \notin A 2^{t}+1 \notin A$ for all $t \geq 0.7 \in A$ implies 4, $6 \in A$ and $G_{2}=(4,6,7) \subset A$. Let $m$ be the maximal number of $m$ such that $G_{m} \subset$ $A, m \geq 2$. Now assume that $G_{m} \neq A$. Then, by (1.4), there exists an integer $n$ such that $2 n \in A$ and $2 n \geq 2^{m+1}$. By Lemma $2.4, A / 2$ is simple and $2 \in A / 2$. Then by Theorem $4.1, A / 2=A_{l}=(2,3, \cdots \cdots, l+1)$ for some $l \neq 2^{m}-1$. Since $n \in A / 2$ and $n \geq$ $2^{m}, A / 2$ contains $2^{m}+1$, hence $2^{m+1}+2 \in A$. Since $2^{m+1}-1 \in G_{m} \subset A$, we have $2^{m+2}-3$ $\in A$ by Lemma 5.3 of $t=m+1, s=1$, and hence $2^{m+2}-1 \in A$ by Lemma 5.4 . Then $2^{m+2}-2 \in A, 2^{m+1}-1 \in A / 2$ and $l+1 \geq 2^{m+1}-1$. Thus $2^{m}+2^{s-1} \in A / 2$ and $2^{m+1}+2^{s} \in A$ for $m>s \geq 1$. Again by use of Lemma 5.3 of $t=m+1$, we have $2^{m+2}-2^{s+1}+1 \in A$ for $m>s \geq 1$ and also for $s=0$ as above. Then it follows from (1.9) $G_{m_{+1}} \subset A$ contradicting the maximality of $m$. Thus we have proved $A=G_{m}$ for some $m \geq 2$.

Case 2: $7 \notin A$. By use of (1.8), we have $2^{r}+3 \notin A$ for $r \geq 2$. By (1.11), there exists some $t$ with $2^{t+1}-1 \in A$, where $t \geq 3$ since $7 \notin A$. We choose the maximal $t$ of $2^{t+1}-1 \in A$. By a similar argument to the case we see $2^{t}+2 \in A$. Then by Lemma $5.52^{t+1}+2 \in A$, and by Lemmas $5.3,5.42^{t+2}-3 \in A$ and $2^{t+2}-1 \in A$ contradicting to the choice of $t$. Thus the case $7 \notin A$ does not occur, and the Theorem 5.1 has been established.

By (1.4) and (1.5), $4 \notin A$ implies $5,6,7 \notin A$. Then by $(1.8) 9,10,11 \notin A$, and $11 \notin A$ implies $13 \notin A$ by (1.5). Again using (1.8) repeatedly we have

(5.1). $4 \notin A$ implies $2^{t}+i \notin A$ for $i=1,2,3,5$ and $t \geq 2$.

Lemma 5.6. Let $t \geq 4$. If an allowable sequence $A$ satisifies $4 \notin A, 2^{t}-1 \in A$ and $2^{t}+6 \in A$ then $2^{t+1}-3 \in A$ and $2^{t+1}-1 \in A$.

Proof. First assume $2^{t+1}-3 \notin A$. Then by (3.4) $S q^{2 t} x_{2} t_{-1}=x_{2} t_{-2} x_{2} t_{-1}+a_{1} x_{2} t_{-3} x_{2} t$ $+a_{5} x_{2} t_{-7} x_{2} t_{+4}+a_{7} x_{2} t_{-9} x_{2} t_{+6}+\cdots \cdots$. Since $2^{t}+3 \notin A$ by (5.1) we have $S q^{8} S q^{2 t-2} x_{2} t_{-1}=0$ by (4.1). Then we have a contradiction: $0=\left\{\left(\begin{array}{c}2^{t}-3 \\ 8\end{array}\right)+a_{1}\left(\begin{array}{c}2^{t}-4 \\ 2\end{array}\right)+a_{5}\left(\begin{array}{c}2^{t}-8 \\ 6\end{array}\right)+a_{7}\left(\begin{array}{c}2^{t}-10 \\ 8\end{array}\right)\right\}$ $x_{2^{t}-1} x_{2^{t}+6}+\cdots \cdots=x_{2^{t}-1} x_{2^{t}+6}+\cdots \cdots$. Thus $2^{t+1}-3 \in A$. Next assume $2^{t+1}-1 \notin A$. As in the proof of Lemma 5.4, we have $S q^{2 t-1} x_{2^{t}}=x_{2^{t}-1} x_{2^{t}}+a_{4} x_{2^{t}-5^{2}} x_{2^{t}+4}+\cdots \cdots$. Since $2^{t}$ $+2 \notin A$ by (5.1), we have $S q^{4} S q^{2 t-1} x_{2^{t}}=0$. Then we have a contradiction: $0=$ $\left\{\left(2^{t}-1\right)+a_{4}\left(2^{t}-6\right)\right\} x_{2^{t}-1} x_{2} t_{+4}+\cdots \cdots=x_{2^{t}-1} x_{2^{t}+4}+\cdots \cdots$, where $2^{t}+4 \in A$ since $2^{t}+6 \in A$. Thus $2^{t+1}-1 \in A$.

Lemma 5.7. Let $t \geq 3$. If an allowable sequence $A$ satisfies $4 \notin A, 2^{t}+4 \in A$ and $2^{t+1}-1 \in A$ then $2^{t}+7 \in A$ or $2^{t+1}+4 \in A$.

Proof. Assume $2^{t}+7 \notin A$ and $2^{t+1}+4 \notin A$. By a similar argument to the proof 
of Lemma 5.5, we have $S q^{2 t} x_{2^{t}+4}=x_{8} x_{2^{t+1}-4}$ tother terms. Then $S q^{3} S q^{2 t} x_{2^{t}+4}=x_{8} x_{2}{ }^{t+1}-1$ tother terms. By Adem relation $S q^{3} S q^{2 t}=S q^{4} S q^{2 t-1}+S q^{2} S q^{2 t-1} S q^{2}+S q^{2 t} S q^{3}$. Then by use of (5.1) and the assumption $2^{t}+7^{t} \notin A, S q^{3} S q^{2} x_{2^{t}+4} \in S q^{4} D^{2}+S q^{2} D^{2}+S q^{2 t} D^{2}$. Thus at least one of $x_{8} S q^{4} x_{2^{t+1}-5}=\left(\begin{array}{c}2^{t+1}-6 \\ 4\end{array}\right) x_{8} x_{2^{t+1}-1}, \quad x_{8} S q^{2} x_{2} t_{+1-3}=\left(\begin{array}{c}2^{t+1}-4 \\ 2\end{array}\right) x_{8} x_{2 t+1-1}$ and $x_{8}$ $S q^{2 t} x_{2^{t}-1}=0$ coincides with $x_{8} x_{2^{t+1}-1}$. But these coefficients are zero modulo 2, and we get a contradiction. Therefore $2^{t}+7 \in A$ or $2^{t+1}+4 \in A$.

Lemma 5.8. Let $t \geq 4$. If an allowable sequence $A$ satisfies $4 \notin A, 2^{t}+4 \in A$ and $2^{t+1}-3 \in A$ then $2^{t+1}+4 \in A$.

Proof. Assume $2^{t+1}+4 \notin A$, then as above we have $S q^{2 t} x_{2^{t}+4}=x_{8} x_{2^{t+1}-4}+$ other terms and $S q^{1} S q^{2 t} x_{2^{t}+4}=x_{8} x_{2^{t+1}-3}$ tother terms. By Adem relation $S q^{1} S q^{2 t}=S q^{2 t+1}=$ $S q^{4} S q^{2 t-3}+S q^{2 t-1} S q^{2}+S q^{2 t} S q^{1}=S q^{4} S q^{2 t-3}+S q^{2 t-1-1} S q^{2 t-1} S q^{2}+S q^{2 t} S q^{1}$. By (5.1) and Lemma $1.2 S q^{2 t-3} x_{2^{t}+4}$ and $S q^{1} x_{2^{t}+4}$ are decomposable. Also, $S q^{2 t-1} S q^{2} x_{2} t_{+4} \equiv S q^{2 t-1} x_{2^{t}+6} \equiv$ $\left(\begin{array}{c}2^{t}+5 \\ 2^{t-1}\end{array}\right) x_{2^{t}+2^{t-1}+6} \equiv 0 \bmod D^{2}$. Thus $S q^{1} S q^{2 t} x_{2^{t}+4} \in S q^{4} D^{2}+S q^{2 t-1-1} D^{2}+S q^{2 t} D^{2}$, and $x_{8} x_{2} 2^{t+1}-3$ coincides at least one of $x_{8} S q^{4} x_{2^{t+1}-7}=\left(\begin{array}{c}2^{t+1}-8 \\ 4\end{array}\right) x_{8} x_{2^{t+1}-3}=0, \quad x_{8} S q^{2 t-1-1} x_{2^{t}+2^{t-1}-2}=$ $\left(\begin{array}{c}2^{t}+2^{t-1}-3 \\ 2^{t-1}-1\end{array}\right) x_{8} x_{2^{t+1}-1}=0$ and $x_{8} S q^{2 t} x_{2} t_{-3}=0$ which is a contradiction. Therefore we have $2^{t+1}+4 \in A$.

Proof of Theorem 5.2.

Case 1: $8 \in A$. By Lemma 2.4, $A / 2$ is simple and $2 \notin A / 2,4 \in A / 2$. It follows from Theorem $5.1 A / 2=G_{m}$ for some $m \geq 2$. Thus $2 G_{m} \subset A$ and $A-2 G_{m}$ consists of odd integers. By (1.11), we have that there exists an integer $s$ such that $2^{s}-1 \in A$ and $A$ does not contain odd integers smaller than $2^{s}-1$. By (1.4), $2^{s}-2 \in A$ and $2^{s-1}-1 \in A / 2=G_{m}$. Then we have $3 \leq s-1 \leq m+1$ and $4 \leq s \leq m+2$. Since $2^{t-1}+2$, $2^{t-1}+3 \in G_{m}=A / 2$ for $3 \leq t \leq m+1$ we have $2^{t}+4,2^{t}+6 \in A$ for $3 \leq t \leq m+1$. Now assume that $4 \leq s<m+2$. Using Lemma 5.6 for $t=s, \cdots \cdots, m+1$ we have from $2^{s}-1$ $\in A$ that $2^{m+2}-3 \in A$. Then, by Lemma $5.8,2^{m+2}+4 \in A$ which contradicts to $2^{m+1}$ $+2 \notin G_{m}=A / 2$. Therefore we must have $s=m+2$. By (1.4) and by the structure of $G_{m}=A / 2, A$ does not contain any integer greater than $2^{m+2}-1$. Thus $A=2 G_{m} \cup$ $\left(2^{m+2}-1\right)$. Next, by Lemma $5.7,2^{m+2}-1 \in A$ implies $2^{m+1}+7 \in A$ or $2^{m+2}+4 \in A$, but $2^{m+2}+4 \notin A$ as above. Thus $2^{m+1}+7 \in A$, hence $2^{m+1}+7=2^{m+2}-1$. It follows that $m$ $=2$ and $A=2 G_{2} \cup(15)=(8,12,14,15)=E_{3}$.

Case 2: $8 \notin A$. By (1.7), $a_{1}=2^{n}$ for some $n$. Since 1, 2, 4, $8 \notin A, n \geq 4$. We shall prove by induction on $n \geq 4$ and assume the theorem is true for $n-1$, i.e., $A / 2=E_{n-1}$. Then $2 E_{n-1} \subset A$ and $A-2 E_{n-1}$ consist of some odd integers $i$ of $i-1 \in$ $2 E_{n-1}$, i.e., $i=2^{n+1}-2^{s}+1$ for some $s$ of $n \geq s \geq 1$. Since $\left(\frac{2^{n+1}-2^{s}-2^{s-1}}{2^{s-1}}\right) \equiv 1,2^{n+1}-2^{s}$ $+1 \in A$ implies $2^{n+1}-2^{s}-2^{s-1}+1 \in A$. This shows that $s$ must be 1 , and we have $A=2 E_{n-1} \cup\left(2^{n+1}-1\right)=\left(2^{n+1}-2^{s} ; n \geq s \geq 0\right)=E_{n}$. This completes the proof of Theorem 5.2 .

\section{Allowability of the sequences $\boldsymbol{G}_{m}$ and $\boldsymbol{E}_{n}$.}

In order to complete the proof of our main theorem we shall prove that the 
sequences $T_{1}, A_{l}, G_{m}$ and $E_{n}$ in the theorem are allowable and simple. By Lemma 2.3, these sequences are simple if it is allowable. We have well-known examples $P\left(T_{1}\right)=H^{*}\left(R P^{\infty} ; Z_{2}\right)$ and $P\left(A_{l}\right)=H^{*}\left(B S O(l+1) ; Z_{2}\right)=Z_{2}\left[w_{2}, w_{3}, \cdots \ldots, w_{l}\right]$ where $w_{i}$ denotes the $i$-th Stiefel-Whitney class. Thus we have

(6.1). $T_{1}=(1)$ and $A=(2,3, \cdots \cdots, l+1), l+1$ is not a power of 2 , are strictly allowable hence allowable and simple.

The Stiefel-Whitney classes $w_{i}$ satisfy the following $\mathrm{Wu}$ formula

(6.2). $S q^{j} w_{i}=\sum_{0 \leq k \leq j}\left(\begin{array}{c}i-j+k-1 \\ k\end{array}\right) w_{j-k} w_{i+k}, w_{0}=1, w_{1}=0$.

The following statements are probably well-known.

(6.3). (i). $\left(\begin{array}{c}2^{t}-k-1 \\ k\end{array}\right) \equiv 1(\bmod 2)$ if and only if $k=0$.

(ii). $\left(\begin{array}{c}2^{t}-2^{s}-k-1 \\ k\end{array}\right) \equiv 1(\bmod 2)$ if and only if $k=2^{q}-2^{s}$ for $s \leq q<t$.

In fact, if $k=2^{r}(2 a+1), a \geq 0$, then $\left(\begin{array}{c}2^{t}-k-1 \\ k\end{array}\right) \equiv\left(\begin{array}{c}2^{t}-2^{r+1}(a+1) \\ 2^{r+1} a\end{array}\right)\left(\begin{array}{c}2^{r}-1 \\ 2^{r}\end{array}\right) \equiv 0, \quad$ and we obtain (i). If $r \neq s$ we have similarly $\left(\begin{array}{c}2^{t}-2^{s}-k-1 \\ k\end{array}\right) \equiv 0$. If $r=s$, we can put $k=2^{q}(2 b+1)-2^{s}, s \leq q<t$. Then $\left(\begin{array}{c}2^{t}-2^{s}-k-1 \\ k\end{array}\right) \equiv\left(\begin{array}{c}2^{t}-2^{q+1}(b+1) \\ 2^{q+1} b\end{array}\right)\left(\begin{array}{c}2^{q}-1 \\ 2^{q}-2^{s}\end{array}\right) \equiv\left(\begin{array}{c}2^{t-q-1}-b-1 \\ b\end{array}\right)$ $\equiv 1$ if and only if $b=0$, by (i), i.e., $k=2^{q}-2^{s}$.

Now consider the squaring operations on $H^{*}\left(B S O\left(2^{n+1}-1\right) ; Z_{2}\right)=Z_{2}\left[w_{2}, w_{3}, \cdots \cdots\right.$, $\left.w_{2^{n+1}-1}\right], n \geq 1$. Let $I$ be the ideal generated by $\left\{w_{i}, i \notin E_{n}\right\}\left(E_{1}=A_{2}, E_{2}=G_{2}\right)$.

Theorem 6.1. $H^{*}\left(B S O\left(2^{n+1}-1\right), Z_{2}\right) / I=P\left(E_{n}\right), n \geq 1$, admits squaring operations, hence $E_{n}\left(E_{1}=A_{2}, E_{2}=G_{2}\right)$ is strictly allowable and it is allowable and simple.

Proof. It is sufficient to prove that $I$ is closed under the squaring operations, or by Cartan formula to prove $S q^{j} w_{i} \in I$ for $i \notin E_{n}$. Assume that

$$
j-k=2^{n+1}-2^{r}, i+k=2^{n+1}-2^{s} \text { and }\left(\begin{array}{c}
i-j+k-1 \\
k
\end{array}\right) \equiv 1(\bmod 2)
$$

for some $i, j, k$ and $0 \leq r \leq n+1,0 \leq s<n+1$. Since $i-j+k-1=2^{r}-2^{s}-k-1$ it follows by (6.3), (ii) that $k=2^{q}-2^{s}$ for some $s \leq q<r$. Thus $i=2^{n+1}-2^{q}, 0 \leq q \leq n$. This shows, by (6.2), that $S q^{j} w_{i} \in I$ for $i \notin E_{n}$, completing the proof.

Next consider $H^{*}\left(B S O\left(2^{m+1}+1\right) ; Z_{2}\right) / D^{3}=Z_{2}\left[w_{2}, w_{3}, \cdots \cdots, w_{2}{ }^{m+1}+1\right] / D^{3}=T P\left(A_{2} m+1\right)$. Define $Q_{k}, k \geq 0$, inductively by

$$
Q_{0}=S q^{0} \text { and } Q_{k}=S q^{2^{k-1}} Q_{k-1} \text { for } k>0 .
$$

By (6.2), $S q^{2 t} w_{2} t+1 \equiv w_{2^{t+1}+1} \bmod D^{2}$. Then by induction on $k$ we have

(6.4). $\quad Q_{k} w_{2} \equiv w_{2^{k}+1} \bmod D^{2}$ for $k \geq 0$.

Let $I^{\prime}$ be the ideal generated by $Q_{k} w_{2}, k \geq 0$. Then it follows from (6.4) that $T P\left(A_{2} m_{+1}\right) / I^{\prime}$ is isomorphic to $T P\left(G_{m} \cup\left(2^{m+1}\right)\right)$. Here remark that $Q_{k} w_{2}=0$, for $k>$ $m+2$ by dimensional reason and $Q_{m+2} w_{2}=w_{2^{m+1}} w_{2^{m+1}+1}=w_{2}{ }^{m+1} Q_{m+1} w_{2}$.

Lemma 6.2. $\left(H^{*}\left(B S O\left(2^{m+1}+1\right) ; Z_{2}\right) / D^{3}\right) / I^{\prime}=T P\left(G_{m} \cup\left(2^{m+1}\right)\right)$ admits squaring operations, hence $G_{m} \cup\left(2^{m+1}\right)$ is allowable.

Proof. It is sufficient to prove

$S q^{i} Q_{k} w_{2} \in I^{\prime}$ for $i>0$.

By (1.1), (iv), $S q^{i} Q_{k} w_{2}=0$ for $i>2^{k}+1, S q^{2 k+1} Q_{k} w_{2}=\left(Q_{k} w_{2}\right)^{2}$ and by definition $S q^{2 k} Q_{k} w_{2}$ 
$=Q_{k} w_{2}$. Then it is sufficient to prove $\left(^{*}\right)$ for $0<i<2^{k}$ by induction on $k$. The case $k=0$ is trivial. By Adem relation, $S q^{i} Q_{k_{+1}} w_{2}=S q^{i} S q^{2 k} Q_{k} w_{2}$ is a linear combination

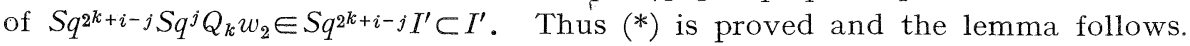

Applying Lemma 2.1 to the result of Lemma 6.2 we have

Theorem 6.3. $G_{m}$ is allowable and simple for $m \geq 1\left(G_{1}=A_{2}\right)$.

Proof of Main Theorem. Let $A$ be a simple sequence. By (1.7), $a_{1}=2^{t}$ for some $t \geq 0$. Then $A=T_{1}, A_{l}, G_{m}, E_{n}$ according to $t=0,1,2, t \geq 3$ by Theorems 4.1, 5.1, $5.2(l+1$ is not a power of 2$)$. Conversely these sequences are simple by (6.1) and Theorems 6.1, 6.2.

Proof of Corollary to Main Theorem. By Theorem (1.1) of [4], $T P(A)=Z_{2}\left[x_{j}\right]$ / $D^{3}, \iota x_{j}=u_{j}$, admits squaring operations. Then the corollary follows from the main theorem.

\section{Bibliography}

[1] Adem, J., The relations on Steenrod powers of cohomology classes, Algebraic Geometry and Topology, Princeton, 1957, 191-238.

[2] Borel, A., Sur la cohomologie des espaces fibrés principaux et des espaces homogènes de groupes de Lie compacts, Ann. of Math. 57 (1953), 115-207.

[3] Borel, A., Sur l'homologie et la cohomologie des groupes de Lie compacts connexes, Amer. J. Math. 76 (1954), 273-342.

[4] Browder, W. and E. Thomas, On the projective plane of an H-space, Illinois J. Math. 7 (1963), 492-502.

[5] Milnor, J. and J.C. Moore, On the structure of Hopf algebras, Ann. of Math. 81 (1965), 211-264.

[6] Steenrod, N. E., Cohomology operations, Ann. of Math. Studies, No. 50, Princeton.

[7] Thomas, E., Steenrod squares and H-spaces: II, Ann. of Math. 81 (1965), 473-495. 